Revue d'histoire du XIXe siècle

Société d'histoire de la révolution de 1848 et des

révolutions du XIXe siècle

Religion, politique et culture au XIXe siècle

\title{
Anne-Emmanuelle Demartini, L'Affaire Lacenaire, Paris, Éditions Aubier, 2001, 430 p.
}

Jean-Jacques Yvorel

\section{OpenEdition}

\section{Journals}

Édition électronique

URL : http://journals.openedition.org/rh19/641

DOI : 10.4000/rh19.641

ISSN : $1777-5329$

\section{Éditeur}

La Société de 1848

\section{Édition imprimée}

Date de publication : 1 juin 2004

Pagination : 195-198

ISSN : 1265-1354

Référence électronique

Jean-Jacques Yvorel, «Anne-Emmanuelle Demartini, L'Affaire Lacenaire, Paris, Éditions Aubier, 2001

430 p. », Revue d'histoire du XIXe siècle [En ligne], 28 | 2004, mis en ligne le 19 juin 2005, consulté le 22 septembre 2020. URL : http://journals.openedition.org/rh19/641 ; DOI : https://doi.org/10.4000/ rh19.641

Ce document a été généré automatiquement le 22 septembre 2020

Tous droits réservés 


\title{
Anne-Emmanuelle Demartini, L'Affaire Lacenaire, Paris, Éditions Aubier, 2001, 430 p.
}

\author{
Jean-Jacques Yvorel
}

1 L'affaire Lacenaire est ce qu'il est convenu d'appeler une « cause célèbre ». Ces affaires extraordinaires ont tant préoccupé les historiens professionnels ou amateurs que leur narration a fini par constituer un genre. Dans les années 1970, un quart des ouvrages sur l'histoire de la justice était consacré à cet exercice qui représente encore $12 \%$ de la production ${ }^{1}$. Pourtant, le livre d'Anne-Emmanuelle Demartini n'appartient pas véritablement à ce genre ou du moins le renouvelle-t-il radicalement. Elle utilise en effet un fait singulier pour interroger l'imaginaire social de la Monarchie de Juillet.

Le caractère exceptionnel de l'affaire ne réside pas dans la nature du crime. PierreFrançois Lacenaire n'est pas un tueur en série et sa culpabilité bien établie ne nécessite aucune révision posthume. Il est condamné à mort en novembre 1835 pour quelques faux en écriture et pour deux assassinats crapuleux. L'originalité de la cause gît tout entière dans la personnalité de Lacenaire. De son procès à sa mort sur l'échafaud en janvier 1836, ce dandy criminel, poète à ses heures, ne cesse de se mettre en scène. Objet de fascination et de scandale, il subvertit le théâtre judiciaire, détourne la règle du jeu.

3 Dans un premier chapitre intitulé "Une cause célèbre des plus extraordinaires », l'auteure présente l'affaire. (Remarquons que faute d'avoir accès au dossier d'instruction qui a disparu, le "rappel des faits» s'appuie sur les comptes-rendus d'audiences du procès.) Anne-Emmanuelle Demartini rappelle comment, entre le 12 et 14 novembre 1835, un héros de cour d'assise est né et aussi comment, lorsque la scène carcérale de la conciergerie remplace la scène judiciaire, la renommé de Lacenaire ne fait que croître.

4 Viennent ensuite cinq chapitres sur Lacenaire comme figure du monstre. Ils constituent la colonne vertébrale de l'ouvrage et la principale clef de lecture proposée par d'Anne-Emmanuelle Demartini. Rapidement, l'auteure utilise le terme de 
surgissement, Lacenaire est identifié comme un monstre (chapitre II : «L'identification du monstre »). Issu d'un milieu honorable, pourvu d'une instruction supérieure et maitrisant le latin, muni d'une bonne éducation morale puisqu'il est passé par le séminaire, Lacenaire ne peut être compris que comme une effroyable exception. C'est un être hybride, à la fois monstre chaud assoiffé de sang et monstre froid philosophe matérialiste. Mais la tératologie classique a ses limites : «La catégorie du monstre est un principe d'intelligibilité tautologique car en tant qu'exception, par définition, le monstre ne renvoie qu'à lui-même" (p.113). Pour construire une explication satisfaisante, il faut que l'exception devienne révélatrice de son temps mais alors le monstre singulier tend à devenir un nouveau type d'assassin inscrit dans une époque et perd de sa singularité. C'est finalement sur les terrains politique, idéologique, social et culturel que les contemporains de l'affaire ont été chercher un sens à donner à Lacenaire.

5 Lacenaire va donc être analysé comme « Un monstre politique » (chapitre III) : produit d'un régime hybride, la Monarchie de Juillet, cet assassin est vu comme la métaphore monstrueuse de la modernité politique. Lacenaire est aussi «Un montre criminel» (chapitre IV), interprète et chef de file d'un monde du crime qui, se riant de la guillotine, interroge l'efficacité même des institutions punitives. Issu de la bonne bourgeoisie lyonnaise et non du lumpenprolétariat des bas-fonds de la capitale (p. 212), Lacenaire est « Un monstre bourgeois » (chapitre V), un produit non pas de l'ignorance mais d'une culture corruptrice, une remise en cause vivante, pour les légitimistes, du principe même des capacités et de la mobilité sociale. Cette sorte "d'altérité sociale interne " conduit les contemporains de Lacenaire à l'identifier aussi sous "les traits d'un monstre démocratique » (p. 212). Enfin, le poète-assassin est "Un monstre romantique » (chapitre VI). Un parallèle est établi entre cette littérature - qui se réclame des passions et glorifie bien des dérèglements comportementaux - et la carrière criminelle de Lacenaire même si ce dernier ne se réclame jamais du romantisme. Parallèle d'autant plus évident que Lacenaire, à la fois dandy, révolté et poète, conjugue trois figures emblématiques du romantisme. À une époque où la littérature « est créditée du pouvoir de produire le social » (p. 239), elle est rapidement accusée « d'avoir permis à Lacenaire de construire sa singularité criminelle » (p. 240).

6 Anne-Emanuelle Demartini renoue ensuite pour trois chapitres avec la chronologie. De sa condamnation à sa mort, la société mène "Le combat contre le monstre " (chapitre VII). Elle s'efforce d'obtenir de Lacenaire « le regret des crimes et l'allégeance aux lois divines ». Devant l'échec de cette stratégie de réintégration du monstre, c'est à la mort «qu'il revient en dernière instance de fixer définitivement le statut de Lacenaire... monstre ou pas? À la guillotine de trancher » (p. 258). Aussi « La mort du monstre » (chapitre VIII) est-elle un enjeu important. Certains n'hésitent pas à falsifier le déroulement de l'exécution et s'efforcent de le faire mourir en lâche. Lacenaire exécuté, le travail de mise en scène du criminel ne cesse pas (chapitre IX : «L'atelier du portrait posthume »). On recherche sur son corps à coups de scalpels et sur le moulage de son crâne, «le vrai » Lacenaire. Ceux qui l'ont croisé (notamment Arago et Reffay de Lusignan) rapportent leurs souvenirs. Enfin on censure et corrige ses mémoires avant de les publier.

7 Dans un ultime chapitre («Lacenaire de la vie au mythe »), l'auteure se livre à une brève analyse des Mémoires de Lacenaire avant d'étudier la postérité littéraire et artistique du personnage. 
8 niveaux de lecture.

C'est une importante contribution à l'histoire du crime et des peurs sociales. Tout comme le communisme, le voleur-assassin est un spectre qui hante le XIX siècle bourgeois. Ceux qui fréquentent les archives judiciaires savent que l'association du vol et de la violence meurtrière est l'acmé de l'horreur bien devant les crimes sexuels qui mobilisent nos angoisses contemporaines et génèrent, aujourd'hui, les peines les plus lourdes. Lacenaire, «figure inaugurale et symbolique du voleur assassin» (p. 350), est un moment clef de la construction de cet avatar des figures de l'effroi ; c'est un phare qui éclaire la peur d'une criminalité nouvelle engendrée par la nouvelle organisation socio-économique et les transformations urbaines.

En déconstruisant le processus de fabrication d'un monstre, Anne-Emmanuelle Demartini met au jour un certain nombre d'invariants, « de véritables constantes de l'imaginaire de la monstruosité »(p.350). Elle offre ainsi une grille d'analyse au sociologue du temps présent qui se penche sur nos « monstres» actuels.

11 L'Affaire Lacenaire nous montre l'intérêt de l'approche micro-historique d'un trajet singulier. Les allers-retours entre l'individu et le social ont une riche portée heuristique et nous pouvons contempler dans le «miroir Lacenaire » le visage inquiet d'une société post-révolutionnaire «écartelée entre un passé révolu et un avenir à fonder » (p. 351). Reste que Lacenaire, l'auteure le reconnaît, garde une grande part de son mystère : il a plus éclairé le $\mathrm{XIX}^{\mathrm{e}}$ siècle que le $\mathrm{XIX}^{\mathrm{e}}$ siècle (ou même le $\mathrm{XX}^{\mathrm{e}}$ siècle) ne l'a éclairé. "L'âme se cache à tous les yeux » écrit Lacenaire dans une remarque très pascalienne à moins qu'elle ne renvoie à quelque illusion biographique plus contemporaine.

\section{NOTES}

1.. Jean-Claude FARCY, L'Histoire de la justice française de la Révolution à nos jours, Paris, Presses universitaires de France, 2001, p. 37. 